\title{
Detection of Legionella pneumophila serogroup 1 in blood cultures from a patient treated with tumor necrosis factor-alpha inhibitor
}

Norihito Kaku ${ }^{1,3}$, Katsunori Yanagihara ${ }^{3,4}$, Yoshitomo Morinaga ${ }^{3,4}$, Tsuyoshi Sato ${ }^{1}$, Munetoshi Nakashima ${ }^{1}$, Takahiro Sakai ${ }^{2}$, Hiroo Tominaga ${ }^{2}$, Fumiko Wakigawa ${ }^{2}$, Seiji Nagashima $^{1}$, Minoru Fukuda ${ }^{1}$, Kohji Hashiguchi ${ }^{1}$, and Shigeru Kohno ${ }^{4}$

${ }^{1}$ Department of Internal Medicine, Japanese Red Cross Nagasaki Genbaku Hospital, Nagasaki, Japan

${ }^{2}$ Department of Clinical Laboratory, Japanese Red Cross Nagasaki Genbaku Hospital, Nagasaki, Japan

${ }^{3}$ Second Department of Internal Medicine, Nagasaki University Graduate School of Biomedical Sciences, Nagasaki, Japan

${ }^{4}$ Department of Laboratory Medicine, Nagasaki University Graduate School of Biomedical Sciences, Nagasaki, Japan

Corresponding author:

Katsunori Yanagihara

Department of Laboratory Medicine

Nagasaki University Graduate School of Biomedical Sciences

1-7-1 Sakamoto, Nagasaki 852-8501, Japan

Tel: +81-95-819-7418; Fax: +81-95-819-7257

E-mai1: k-yanagi@nagasaki-u.ac.jp 


\begin{abstract}
A 65-year-old man was admitted to our hospital with a fever of $39.3^{\circ} \mathrm{C}$, cough, sputum and pharyngeal discomfort that had persisted for three days. Rheumatoid arthritis had
\end{abstract} been treated with methotrexate and adalimumab for two years and pancreatic metastasis of gastric cancer had been treated with S-1 (tegafur, gimeracil, and oteracil potassium) for two months. Regardless of the underlying pathologies, his general condition was good and he had worked as an electrician until two days before admission. However, his appetite had suddenly decreased from the day before admission, and high fever and hypoxia were also evident upon admission. A chest X-ray and computed tomography scan revealed left pleural effusion and consolidation in both lungs. The pneumonia severity index score was 165 and risk class was V. Accordingly, we started to treat the pneumonia with a combination of levofloxacin and meropenem. Thereafter, we received positive urinary antigen test (UAT) findings for Legionella pneumophila. Despite the application of appropriate antibiotics, vasopressors and oxygenation, the hypotension and hypoxia progressed and the patient died eight hours after admission. Even after his death, blood cultures had been continued to consider the possibility of bacterial co-infection. Although no bacteria were detected from blood cultures, Gimenez staining 
revealed pink bacteria in blood culture fluids. Subsequent blood fluid culture in selective medium revealed L. pneumophila serogroup 1 .

Key words: Legionnaires’ disease, community-acquired pneumonia, blood culture, tumor necrosis factor-alpha inhibitor 


\section{INTRODUCTION}

Since the first outbreak of Legionnaires’ disease (LD) at the American Legion convention in 1976 [1], Legionella pneumophila has been a relatively common pathogen of community-acquired pneumonia (CAP). The reported incidence of LD in CAP ranges from $1.6 \%$ to $7.5 \%$ [2-6] and that in severe CAP is between $14 \%$ and 22.8\%) [6-8]. Legionnaires’ disease has appeared in hospitals, long-term care facilities and other types of accommodation [9-15], causing serious problems. Rapid diagnosis is important to prevent LD outbreaks. Among diagnostic tests for LD, the urinary antigen test (UAT) is the most useful [15] and it supposedly improves the outcomes and mortality rates of patients involved in LD outbreaks [16]. However, detection of the organism by culture is still important, because UAT can detect only L. pneumophila serogroup 1 and approximately 14\% to $20 \%$ of LD is caused by non-serogroup 1 Legionella species $[15,17]$.

In order to use examinations such as UAT effectively, it is important to suspect LD early. Although one review describes clinical clues that increase the possibility of identifying LD [18], to suspect clinical LD remains difficult because clinical 
manifestations are non-specific. Therefore, risk factors for LD are important to

understand. Smoking has been reported as the most common risk factor for LD in some studies [19-21]. However, other recent reports have described LD arising in patients administered with tumor necrosis factor-alpha (TNF- $\alpha$ ) inhibitors [22-25], and these drugs are now recognized as a risk factor for LD [22]. The US Food and Drug Administration (FDA) has updated the indications for the use of TNF- $\alpha$ inhibitors to include risk of Legionella infection [26].

We detected L. pneumophila serogroup 1 in blood cultures from a Japanese patient with severe CAP who had received a TNF- $\alpha$ inhibitor. Although there have been no report of such a case in Japan, it might have to be recognized that Legionella pneumophila might be a pathogen of severe CAP, considering the increased frequency of TNF- $\alpha$ inhibitor use. 


\section{Case report}

A 65-year-old man developed pharyngeal discomfort that persisted for three days. He was admitted to hospital with a fever of $39.3^{\circ} \mathrm{C}$, cough and sputum. He had been treated for rheumatoid arthritis with methotrexate and adalimumab (TNF- $\alpha$ inhibitor) for two years. He had undergone distal gastrectomy to treat gastric cancer four months previously, but pancreatic metastasis discovered two months later resulted in treatment with S-1 (tegafur, gimeracil, and oteracil potassium). He smoked one pack of cigarettes per day, and consumed alcohol socially. There was no history of Hot Springs or travel. Regardless of the underlying pathologies, his general condition was good and he had worked as an electrician until two days before admission. However, his appetite suddenly decreased from the day before admission, when he presented with a temperature of $39.3^{\circ} \mathrm{C}$ and hypoxia $\left(\mathrm{SpO}_{2}, 85 \%\right.$, room air).

Physical findings were normal, except for a slight clouding of consciousness and moist rales in the lower lung field. A chest X-ray and computed tomography (CT) scan revealed left pleural effusion and consolidation accompanied by ground-glass opacity (GGO) in both lungs (Fig. 1). The physical and radiological findings indicated a 
diagnosis of pneumonia. Laboratory data showed a decrease in white blood cells (WBC)

and platelets (PLT) (WBC, $2700 / \mu \mathrm{L}$; PLT, 139,000/ $\mathrm{LL}$ ), renal dysfunction (blood urea nitrogen, $35.1 \mathrm{mg} / \mathrm{dL}$; serum creatinine, $1.5 \mathrm{mg} / \mathrm{dL}$ ), and serum lactic dehydrogenase (LDH) and C-reactive protein (CRP) levels were respectively elevated at $544 \mathrm{U} / \mathrm{L}$ and $31.77 \mathrm{mg} / \mathrm{dL}$. The pneumonia severity index (PSI) for adults [27] was 165 and risk class was V. Accordingly, we started treating the pneumonia with levofloxacin and meropenem. Thereafter, the UAT findings for L. pneumophila were positive. However, the hypotension and hypoxia progressed, and the patient refused intubation due to the underlying diseases. Despite the use of appropriate antibiotics, a vasopressor and oxygenation, the patient died eight hours after admission.

Even after his death, blood cultures had been continued to consider the possibility of bacterial co-infection. The blood cultures appeared negative for co-infective bacteria and Gram staining, but Gimenez staining revealed positive (pink) bacteria (Fig. 2A). Subsequently, L. pneumophila serogroup 1 was detected in the blood culture fluid. The process was as follows. Seven days after starting cultures, the content fluid was withdrawn, separated by centrifugation and applied to Wadowsky-Yee-Okuda (WYO) 
medium, blood agar, bromothymol blue lactose (BTB) agar and chocolate agar media.

Colonies were evident after four days only in WYO medium (Fig. 2B). Gimenez staining again revealed pink bacteria (Fig. 2C), and Gram staining revealed gram-negative rods (Fig. 2D). An antigen-antibody reaction then confirmed the bacteria as L. pneumophila serogroup 1 . 


\section{Discussion}

The two key findings of this case are that L. pneumophila serogroup 1 was detected from blood cultures and that L. pneumophila caused severe CAP in a patient under treatment with a TNF- $\alpha$ inhibitor.

We diagnosed LD using UAT and cultures. The primary method of LD diagnosis is UAT since this modality identifies $81 \%$ to $97 \%$ of LD infections compared to only $5 \%$ to $9 \%$ by culture $[15,28]$. The UAT is simple and it can rapidly and accurately (sensitivity, $60 \%$ to $80 \%$; specificity, >99\%) identify L. pneumophila serogroup 1 [17, 28]. However, culture remains important because $14 \%$ to $20 \%$ of LD infections are caused by non-serogroup 1 Legionella species $[15,17]$ that UAT cannot detect. Notably, L. pneumophila from the present patient survived in a non-selective medium for one week and another study has shown that L. pneumophila could survive in standard blood culture broth medium [29]. Although the number of report of detection of $L$. pneumophila was actually very few [30-32], this case suggests that blood culture is worth trying when UAT results are negative and sputum is not discharged when LD is suspected. 
Diabetes mellitus, current smoker, traveling abroad was reported as risk factors for LD [19-21]. Our patient was current smoker, and it was reported as the most common risk factor for LD [19-21]. Multivariable analysis has identified current cigarette smoking as a dose-dependent risk factor (multivariable odds ratio [OR] for currently smoking >70 cigarettes/week, 13.5; 95\% confidence interval [CI], 5 - 36) [20]. Our patients received S-1. However, the incidence of LD arising in patients under therapy with anti-cancer drugs (such as S-1) does not seem to be increased. A previous study did not find an unusually high incidence of LD among patients with neutropenia, acute leukemia or HIV [33].

Our patients also received TNF-alpha inhibitor. The use of TNF-alpha inhibitor has been reported as a risk factor for serious infections [34]. Recently, some reports have described LD in patients receiving TNF- $\alpha$ inhibitors [22-24] and the relative risk for LD in patients treated with TNF- $\alpha$ inhibitor was between 16.5 and 21 compared to that for the overall population [22]. Although our patients received methotrexate and some reports described that the incidence of serious infections in patients with TNF-alpha inhibitor was not significantly higher than those with disease modifying anti-rheumatic 
drugs (DMARDs) [35, 36], two in vivo studies have reinforced the notion of an increasing risk for LD among patients receiving TNF- $\alpha$ inhibitors. First, the administration of a TNF- $\alpha$-neutralizing antibody to mice in vivo inhibited TNF- $\alpha$ activity and resulted in enhanced L. pneumophila growth in the mouse lung [37]. Secondly, the mortality rates of TNF receptor (TNFR) 1- and TNFR2-knockout mice infected with L. pneumophila [38] are higher than those of wild-type mice, and $L$. pneumophila clearance is slower among TNFR1- than TNFR2-knock-out and wild type mice. That study also indicated that TNF- $\alpha$ is involved in L. pneumophila infection, since L. pneumophila proliferated in peritoneal macrophages, neutrophil accumulation was decreased in bronchoalveolar lavage (BAL) fluids and cytokines (IFN- $\gamma$, interleukin-12 and TNF- $\alpha$ ) were dysregulated in TNFR1-knock-out mice. In contrast, large amounts of neutrophils accumulated in BAL fluids from TNFR2-knock-out mice. These data indicated that a TNFR1 deficiency leads to compromised innate immunity against L. pneumophila, whereas a TNFR2 deficiency induces an excessive inflammatory response.

In conclusion, this case showed that blood culture remains valid for diagnosing LD 
and the risk of LD in patients under treatment with TNF- $\alpha$ inhibitors. Blood culture is worth trying in the absence of other findings if LD is suspected. In addition, $L$. pneumophila should be considered as a causative pathogen if severe pneumonia develops in a patient undergoing treatment with TNF- $\alpha$ inhibitors. 


\section{Figure legends}

Figure 1. Chest X-ray (A) and Computed tomography (CT) (B, C).

Left pleural effusion and consolidation accompanied by ground-glass opacity (GGO) in both lungs evident in all images.

Figure 2. Blood culture fluid staining.

Bacteria in blood culture fluid identified by pink Gimenez staining (A). Four days after starting culture in selective medium, colonies appeared in Wadowsky-Yee-Okuda (WYO) medium, but not in blood agar, bromothymol blue lactose (BTB) agar or chocolate agar media (B). Gimenez stain also stained bacteria from colonies in WYO medium pink (C) and Gram staining revealed gram-negative rods (D). (A), (C) and (D), $\times 1000$ magnification. 


\section{References}

1. Fraser DW, Tsai TR, Orenstein W, Parkin WE, Beecham HJ, Sharrar RG, et al. Legionnaires' disease: description of an epidemic of pneumonia. $N$ Engl J Med. 1977; 297: 1189-97.

2. Fang GD, Fine M, Orloff J, Arisumi D, Yu VL, Kapoor W, et al. New and emerging etiologies for community-acquired pneumonia with implications for therapy. A prospective multicenter study of 359 cases. Medicine (Baltimore). 1990; 69: $307-16$.

3. Marston BJ, Plouffe JF, File TM Jr, Hackman BA, Salstrom SJ, Lipman HB, et al. Incidence of community-acquired pneumonia requiring hospitalization. Results of a population-based active surveillance Study in Ohio. The Community-Based Pneumonia Incidence Study Group. Arch Intern Med. 1997; 157: 1709-18.

4. Lim WS, Macfarlane JT, Boswell TC, Harrison TG, Rose D, Leinonen M, et al. Study of community acquired pneumonia aetiology (SCAPA) in adults admitted to hospital: implications for management guidelines. Thorax. 2001; 56: 296-301.

5. von Baum HS, Ewig S, Marre R, Suttorp N, Gonschior S, Welte T, et al. Community-acquired Legionella pneumonia: new insights from the German competence network for community acquired pneumonia. Clin Infect Dis. 2008;

46: $1356-64$.

6. Falcó V, Fernández de Sevilla T, Alegre J, Ferrer A, Martinez Vázquez JM. Legionella pneumophila: a cause of severe community-acquired pneumonia. Chest. 1991; 100: 1007-11.

7. Torres A, Serra-Batlles J, Ferrer A, Jiménez P, Celis R, Cobo E, et al. Severe community-acquired pneumonia: epidemiology and prognostic factors. Am Rev Respir Dis. 1991; 144: 312-8. 
8. Rello J, Quintana E, Ausina V, Net A, Prats G. A three-year study of severe community-acquired pneumonia with emphasis on outcome. Chest. 1993; 103: 232-5.

9. Marrie TJ, MacDonald S, Clarke K, Haldane D. Nosocomial Legionnaires' disease: Lessons from a four-year prospective study. Am J Infect Control. 1991; 19: 63-6.

10. Sopena N, Sabrià M; the Neunos 2000 Study Group. Multicenter Study of Hospital-Acquired Pneumonia in Non-ICU Patients. Chest. 2005; 127: 213-9.

11. Maesaki S, Kohno S, Koga H, Kaku M, Yoshitomi Y, Yamada H, et al. An outbreak of Legionnaires’ pneumonia in a nursing home. Intern Med. 1992; 31: 508-12.

12. Stout JE, Brennen C, Murder RR. Legionnaires' disease in a newly constructed long-term care facility. J Am Geriatr Soc. 2000; 58: 1589-92.

13. Phares CR, Russell E, Thigpen MC, Service W, Crist MB, Salyers M, et al. Legionnaires' disease among residents of a long-term care facility: the sentinel event in a community outbreak. Am J Infect Control. 2007; 35: 319-23.

14. Centers for Disease Control and Prevention (CDC). Surveillance for travel-associated Legionnaires’ disease_-United States, 2005-2006. MMWR Morb Mortal Wkly Rep. 2007; 56: 1261-3.

15. Joseph CA, Ricketts KD; European Working Group for Legionella Infections. Legionnaires’ disease in Europe 2007-2008. Euro Surveill. 2010; 15: 19693.

16. Alvarez J, Dominguez A, Sabrià M, Ruiz L, Tormer N, Cayla J, et al. Impact of the Legionella urinary test on epidemiological trends in community outbreaks of legionellosis in Catalonia, Spain, 1990-2004. Int J Infect Dis. 2009; 13: e365-70.

17. Fields BS, Benson RF, Besser RE. Legionella and Legionnaires’ disease: 25 years of investigation. Clin Microbiol Rev. 2002; 15: 506-26. 
18. Mulazimoglu L, Yu VL. Can Legionnaires’ disease be diagnosed by clinical criteria? A critical review. Chest. 2001; 120: 1049-53.

19. Greig JE, Carnie JA, Tallis GF, Ryan NJ, Tan AG, Gordon IR, et al. An outbreak of Legionnaires' disease at the Melbourne Aquarium, April 2000: Investigation and case-control studies. Med J Aust. 2000; 180: 566-72.

20. Den Boer JW, Nijhof J, Friesema I. Risk factors for sporadic community-acquired Legionnaires’ disease. A 3- year national case-control study. Public Health. 2006; 120: 566-71.

21. Nakamura S, Yanagihara K, Izumikawa K, Seki M, Kakeya H, Yamamoto Y, et al. The clinical efficacy of fluoroquinolone and macrolide combination therapy compared with single-agent therapy against community-acquired pneumonia caused by Legionella pneumophila. J Infect. 2009; 59: 222-4.

22. Tubach F, Ravaud P, Salmon-Ceron D, Petitpain N, Brocq O, Grados F, et al. Emergence of Legionella pneumophila pneumonia in patients receiving tumor necrosis factor-alpha antagonists. Clin Infect Dis. 2006; 43: e95-100.

23. Jinno S, Pulido S, Plen BC. First reported United States case of Legionella pneumophila serogroup 1 pneumonia in a patient receiving anti-tumor necrosis factor-alpha therapy. Hawaii Med J. 2009; 68: 109-12.

24. Beigel F, Jürgens M, Filic L, Bader L, Lück C, Göke B, et al. Severe Legionella pneumophila pneumonia following infliximab therapy in a patient with Crohn's disease. Inflamm Bowel Dis. 2009; 15: 1240-4.

25. Mariette X, Gottenberg JE, Ravaud P, Combe B. Registries in rheumatoid arthritis and autoimmune diseases: data from the French registries. Rheumatology (Oxford). 2011; 50: 222-9.

26. FDA Drug Safety Communication: drug labels for the tumor necrosis factor-alpha $(\mathrm{TNF} \alpha)$ blockers now include warnings about infection with Legionella and Listeria bacteria. http://www.fda.gov/Drugs/DrugSafety/ucm270849.htm (Accessed on April 16, 2012). 
27. Fine MJ, Auble TE, Yealy DM, Hanusa BH, Weissfeld LA, Singer DE, et al. A prediction rule to low-risk patients with community-acquired pneumonia. $N$ Engl $J$ Med. 1997; 336: 243-50.

28. Centers for Disease Control and Prevention (CDC). Legionellosis - United States, 2000-2009. MMWR Morb Mortal Wkly Rep. 2011; 60: 1083-6.

29. Cirillo DM, Baron EJ, Marchiaro G. Legionella pneumophila fails to multiply in blood culture broths but can be recovered from isolator tubes. Abstr. $100^{\text {th }}$ Gen. Meet. Am. Soc. Microbiol. 2000; Abstr. (C-255): 189.

30. Macrae AD, Greaves PW, Platts P. Isolation of Legionella pneumophila from blood culture. Br Med J. 1979; 2: 1189-90.

31. Rihs JD, Yu VL, Zuravleff JJ, Goetz A, Muder RR. Isolation of Legionella pneumophila from blood with the BACTEC system: a prospective study yielding positive results. J Clin Microbiol. 1985; 22: 422-4.

32. Lai CC, Tan CK, Chou CH, Hsu HL, Huang YT, Liao CH, Hsueh PR. Hospital-acquired pneumonia and bacteremia caused by Legionella pneumophila in an immunocompromised patient. Infection. 2010; 38: 135-7.

33. Sandkovsky U, Sandskovsky G, Suh J, Smith B, Sharp V, Polsky B. Legionella pneumonia and HIV: case reports and review of the literature. AIDS Patient Care STDS. 2008; 22: 473-81.

34. Bongartz T, Sutton AJ, Sweeting MJ, Buchan I, Matteson EL, Montori V. Anti-TNF antibody therapy in rheumatoid arthritis and the risk of serious infections and malignancies: systematic review and meta-analysis of rare harmful effects in randomized controlled trials. JAMA. 2006; 295. 2275-85.

35. Grijalva CG, Chen L, Delzell E, Baddley JW, Beukelman T, Winthrop KL, et al. Initiation of tumor necrosis factor- $\alpha$ antagonists and the risk of hospitalization for infection in patients with autoimmune diseases. JAMA. 2011; 306: 2331-9. 
36. Dewedar AM, Shalaby MA, Al-Homaid S, Mahfouz AM, Shams OA, Fathy A. Lack of adverse effect of anti-tumor necrosis factor- $\alpha$ biologics in treatment of rheumatoid arthritis: 5 years follow-up. Int J Rheum Dis. 2012; 15: 330-5.

37. Brieland JK, Remick DG, Freeman PT, Hurley MC, Fantone JC, Engleberg NC. In vivo regulation of replicative Legionella pneumophila lung infection by endogenous tumor necrosis factor-alpha and nitric oxide. Infect Immun. 1995; 63: 3253-8.

38. Fujita M, Ikegame S, Harada E, Ouchi H, Inoshima I, Watanabe K, et al. TNF receptor 1 and 2 contribute in different ways to resistance to Legionella pneumophila-induced mortality in mice. Cytokine. 2008; 44: 298-303. 
Figure 1.
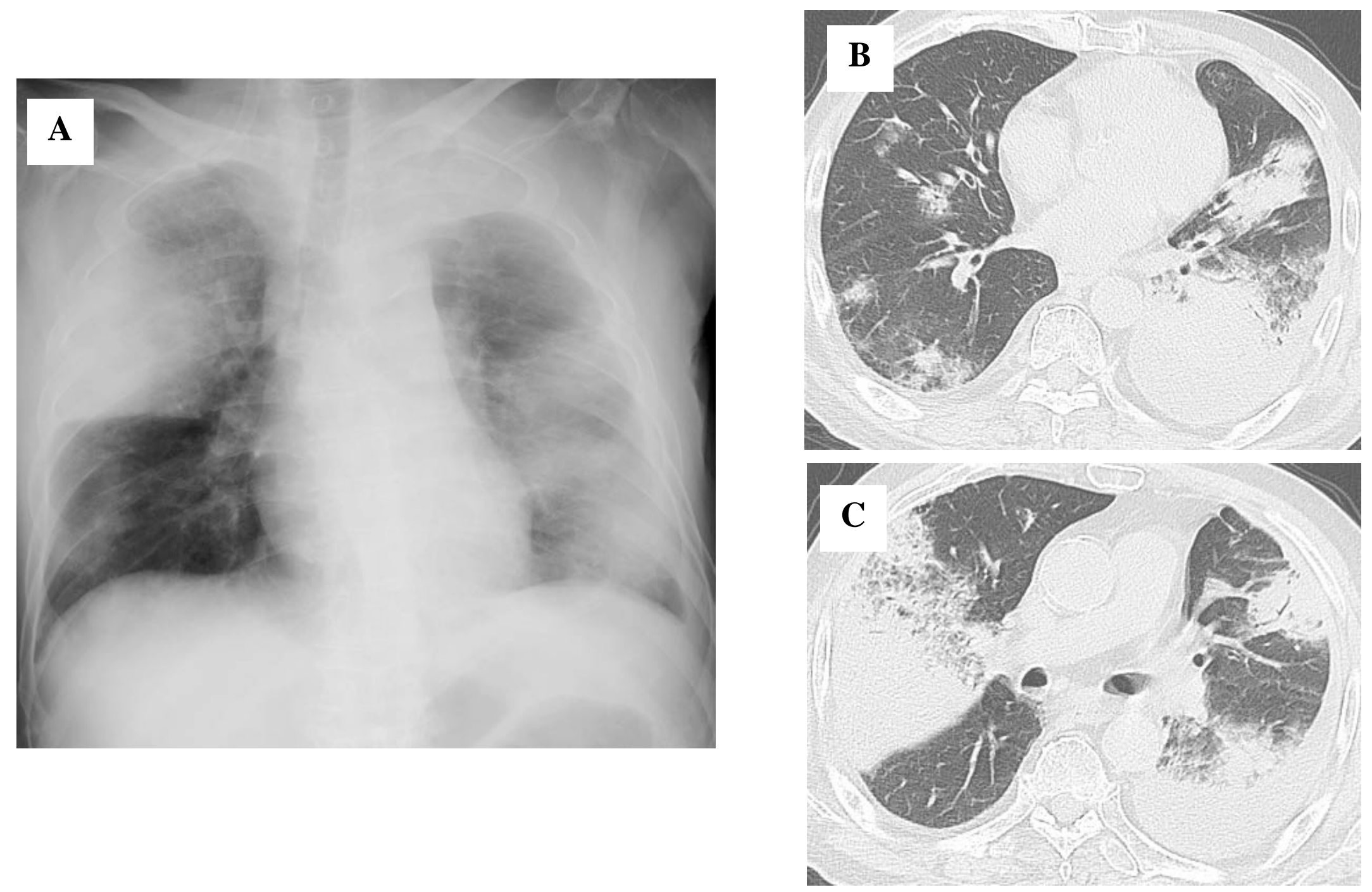
Figure 2.
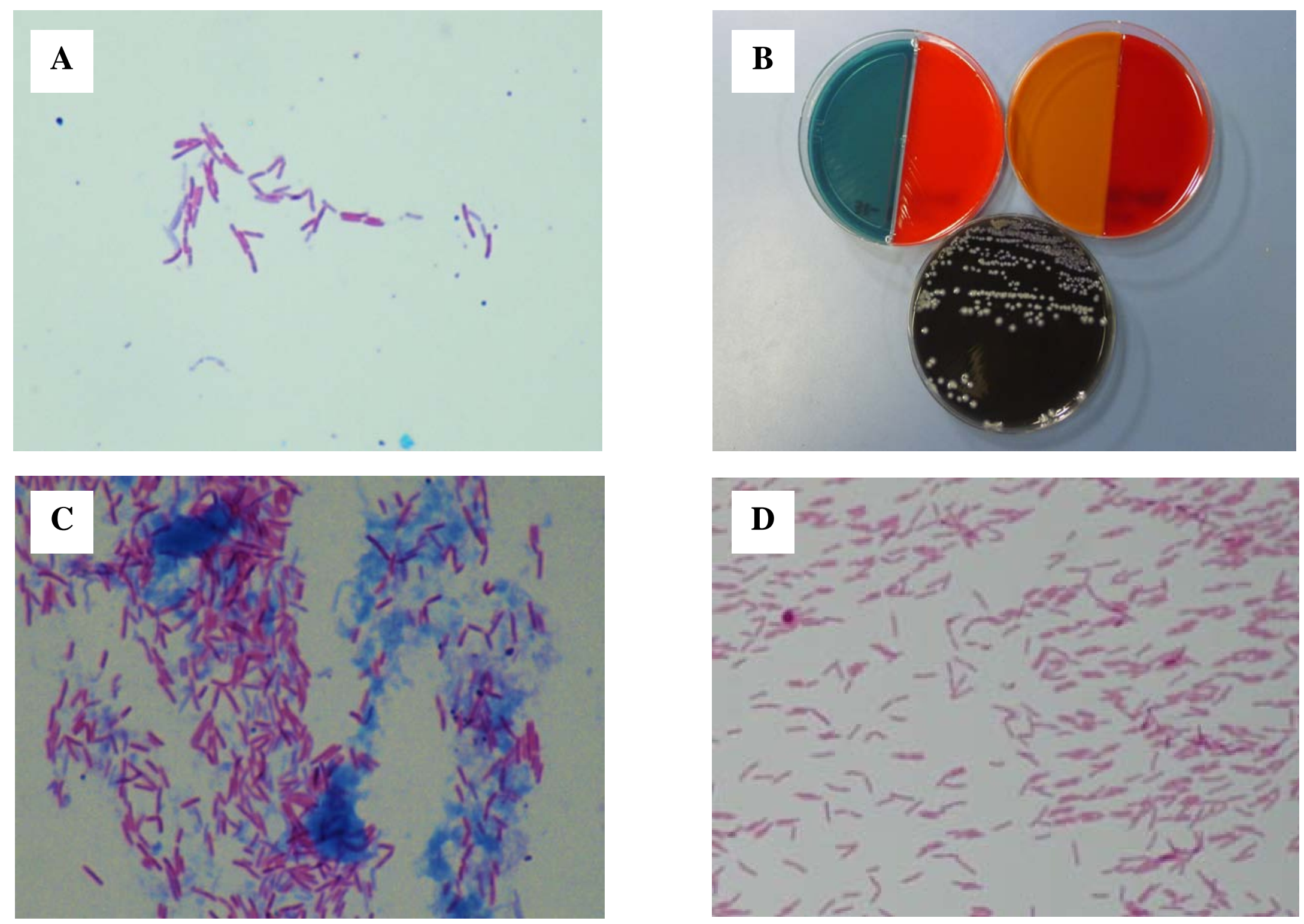